\title{
Monte Carlo Simulation Of The Portfolio-Balance Model Of Exchange Rates: Finite Sample Properties Of The GMM Estimator
}

Hong-Ghi Min, (Email: hmin@icu.ac.kr), Information and Communications University, Republic of Korea

\begin{abstract}
Using Monte Carlo simulation of the Portfolio-balance model of the exchange rates, we report finite sample properties of the GMM estimator for testing over-identifying restrictions in the simultaneous equations model. F-form of Sargan's statistic performs better than its chi-squared form while Hansen's GMM statistic has the smallest bias.
\end{abstract}

\section{INTRODUCTION}

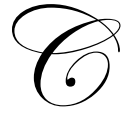

onsistent estimation of linear simultaneous equations model requires minimal set of identifying restrictions in the system and validity of those restrictions can be tested if a model is over-identified. While some statistics are developed and those statistics are widely applied to test the specification of the estimated equations, test the validity of the instrumental variables, and to test if the estimated equations encompass the reduced form little is known the small sample properties of those statistics. For this reason, We investigate the small sample properties of four different over-identifying test statistics for simultaneous equations model including Hausmann(1990)'s Likelihood-ratio test, Sargan(1958, 1960)'s minimum characteristic root statistic, Sargan(1980)'s corresponding F-statistic (Sargan, 1980), and Hansen(1982)'s GMM statistic.

\section{RESPONSE SURFACE METHODOLOGY, TEST STATISTICS, AND THE DGP}

\section{Response Surface Methodology}

To overcome the specificity of Monte Carlo studies to the particular parameter and sample sizes employed, we utilize response surface methodology of Hendrey (1984). Let $\pi_{T}$ be the finite sample probability of the test statistic lying in the critical region. In an experiment the DGP generates $\mathrm{N}$ sets of replications and the statistics lie in the critical region $\mathrm{S}$ out of $\mathrm{N}$ times. An explicit relationship can be defined as equation (7) in section 2.3.

$E(s)=\pi_{T}=f(\theta, T)$

Monte Carlo estimator s gives:

$E(s)=\pi_{T}=f(\theta, T)+\varepsilon, \varepsilon_{i} \sim D\left(0, \pi_{T}\left[1-\pi_{T}\right] / N\right)$

Let $\pi_{a}$ is the analytically calculable asymptotic power of the test and $p^{+}(\theta, T)$ is the discrepancy between the finite sample and asymptotic power, i.e., $T^{-1 / 2}\left\{p\left(\theta, T^{-1 / 2}\right)-q\left(\theta, T^{-1 / 2}\right)\right\}$.

Then,

$p^{+}(\theta, T)=T^{-1 / 2} p\left(\theta, T^{-1 / 2}\right)$

holds. 
$p^{+}\left(\theta, T^{-1 / 2}\right)$ is $O\left(T^{0}\right)$ and is a polynomial in powers of $T^{-1 / 2}$ and the elements of $\theta$.

Using (1)-(3), we can set up a stochastic relationship between a feasible and unbiased estimator of $\pi_{T}$ and the unknown quantity $\pi_{a}, \theta, T$ as equation (4).

$s-\pi_{a}=T^{-1 / 2} q\left(\theta, T^{-1 / 2}\right)+e$

where $q\left(\theta, T^{-1 / 2}\right)$ is an approximation to $p\left(\theta, T^{-1 / 2}\right)$ and the error $\mathrm{e}$ is the combination of $\varepsilon$ and $T^{-1 / 2}\left\{p\left(\theta, T^{-1 / 2}\right)-q\left(\theta, T^{-1 / 2}\right)\right\}$. The choice of $q\left(\theta, T^{-1 / 2}\right)$ is arbitrary but a finite polynomial in $\theta$ and $T^{-1 / 2}$ is common and coefficients on the terms of $q\left(\theta, T^{-1 / 2}\right)$ may be estimated by least squares.

The Test Statistics And Their Asymptotic Properties

We consider four statistics whose statistical descriptions are given in Table 1.

Table 1: Statistics Of Over-Identifying Restrictions

\begin{tabular}{|c|c|c|c|}
\hline \multicolumn{3}{|c|}{ Statistics } & \multirow{2}{*}{$\begin{array}{l}\text { Asymptotic Distributions } \\
\text { (Degrees of freedom) }\end{array}$} \\
\hline Name & Type & Sources & \\
\hline $\mathrm{C}_{0}$ & IV & Sargan (1958) & Chi-squared (n) \\
\hline $\mathrm{F}_{0}$ & IV & Sargan (1980) & Chi-squared with $\left(\mathrm{m}-\mathrm{k}_{0}\right)$ \\
\hline $\mathrm{G}_{0}$ & GMM & Hansen (1983) & $\mathrm{F}\left(\mathrm{m}-\mathrm{k}_{0}, \mathrm{~T}-\mathrm{m}\right)$ \\
\hline $\mathrm{L}_{0}$ & Likelihood- ratio test & Harvey (1990) & Chi-squared(n) \\
\hline
\end{tabular}

(1). $T$ is the econometric sample size, $m$ is the number of instrumental variables, and $k_{i}$ is the number of coefficients estimated under $\mathrm{H}_{\mathrm{i}}$. (2). The statistic $\mathrm{F}_{0}$ is $\left[\mathrm{C}_{0} /\left(\mathrm{m}-\mathrm{k}_{0}\right)\right]^{*}\left[(\mathrm{~T}-\mathrm{m}) /\left(\mathrm{T}-\mathrm{k}_{0}\right)\right] /\left[1-\mathrm{C}_{0} /\left(\mathrm{T}-\mathrm{k}_{0}\right)\right]$ which is $\mathrm{C}_{0} /\left(\mathrm{m}-\mathrm{k}_{0}\right)$ plus finite sample adjustment arising from the finite sample boundedness of $\mathrm{C}_{0}$. (3). $\mathrm{n}$ is the number of over-identifying restrictions in the system.

\section{The Data Generating Process: Portfolio-Balance Model Of Exchange Rates}

True reduced form [equations (7)-(8)] is derived from the true structural form [equation (5)-(6)]. For alternative structural form, $x_{2, t-1}$ and $x_{7, t-1}$ are falsely included into the system [equations (9)-(10)].

$y_{t}=\alpha_{1} Y_{t}+\beta_{1}\left(y_{2, t}-x_{7, t}\right)+\gamma_{1} X_{t}+\varepsilon_{1, t},\left(\varepsilon_{1, t}: \varepsilon_{2, t}\right)^{\prime} \sim N I D(O, \Sigma)$

$Y_{t}=\alpha_{2}+\delta y_{1, t}+\gamma_{2} X_{t}+\varepsilon_{2, t}$

where $\left(y_{t}, Y_{t}\right)^{\prime}$ and $X_{t}$ are $2 \times 1$ and $7 \times 1$ vectors of endogenous and exogenous variables at time $\mathrm{t}(\mathrm{t}=1, \ldots ., \mathrm{T})$; $X_{t}{ }^{\prime}=\left(x_{1 t}, x_{2 t}, x_{3 t}, x_{4 t}, x_{5 t}, x_{6 t}, x_{7 t}\right) ; \beta$ and $\delta$ are simultaneity parameters.

$$
y_{t}=\alpha_{3}+\gamma_{3} X_{t}+\mu_{1 t},\left(\mu_{1 t}: \mu_{2 t}\right)^{\prime}=\Omega
$$

$$
Y_{t}=\alpha_{4}+\gamma_{4} X_{t}+\mu_{2 t}
$$

Alternative structural model is given as equations (9) and (10).

$y_{t}=\alpha_{5}+\beta_{1}\left(y_{2, t}-x_{7, t-1}\right)+\beta_{2}\left(x_{2, t}-x_{2, t-1}\right)+\gamma_{5} X_{t}+\varepsilon_{1, t},\left(\varepsilon_{1, t}: \varepsilon_{2, t}\right)^{\prime} \sim \operatorname{NID}(0, \Sigma)$

$Y_{t}=\alpha_{6}+\delta y_{1, t}+\gamma_{6} X_{t}+\varepsilon_{2, t}$ 
Independent samples of endogenous variables are generated by the population defined in equation (5)-(10) $\dot{a}$ la Basmann (1960). Error terms are generated from linear transformation of $\varepsilon_{i}-\mathrm{N}(0,1)$ and coefficients of transformation are from the Choleski decomposition of the variance-covariance matrices. The fraction of rejections $\mathrm{s}$ $(=\mathrm{S} / 1,000)$ is an unbiased Monte Carlo estimator of the unknown finite sample rejection frequency $\pi_{T}$.

\section{EXPERIMENTAL DESIGN AND COMPUTATIONAL ASPECTS}

The Monte Carlo design variables for the econometric model (7)-(9) are $\theta$ and $T$, where,

$\theta=(\beta, \delta, W, T) \in \Theta=\{\theta|| \Omega|>0 ;| \Sigma \mid>0\}$

and $W=|\Sigma| /|\Omega|, \quad \Sigma$ is the variance-covariance matrix of the reduced form equations and $\Omega$ is the variancecovariance matrix of the structural form equations.

$T \in \mathfrak{I}=\left[T_{a}, T_{b}\right]$

where $T_{a}$ and $T_{b}$ are the smallest and largest econometric sample size considered. For a given statistic $\phi$ (say) and critical value $\delta$, the objective of Monte Carlo study is to find statistics' finite sample rejection frequency $\pi_{T} \equiv \operatorname{prob}(|\phi| \geq \delta)$ for the DGP and those relationships of interest over $\Theta \times \mathfrak{J}$.

$\pi_{T} \equiv \operatorname{prob}(|\phi| \geq \delta \mid \theta, T)=g(\theta, T)$

The value of key parameters $\beta_{1}, \delta, W, T$ cover a range typical of econometric models estimated with actual data: $\beta_{1}=(0.3,0.8), \quad \beta_{2}=2.244, \delta=(0.4,0.7), \mathrm{T}=(20,40,60,80), W=(0.4,0.6,0.8,0.9)$. All other parameters are from reported values of Branson et al (1979). The number of replications is 1,000. Given the choice of parameters, a full factorial design is adopted, resulting in 64 experiments in all. Structural model is estimated by two-stage least squares and Ordinary least squares are used for the reduced form. The asymptotic powers of four statistics were calculated with critical values corresponding to the 5 percent.

\section{POST-SIMULATION ANALYSIS}

This section approximates the finite sample properties of the test statistics by various analytical and numerical-analytical formulae, and examines how well these formulae perform. Response surface regressions are reported in Table 2.

\section{Nominal Size Of Four Statistics}

Response surface of nominal size is reported in the first panel of the Table 2. Most of the estimated coefficients are significant but that of $S_{0}$. Size is well approximated for $\mathrm{G}_{0}$ while $\mathrm{S}_{0}$ is poorly approximated by the sample size and the ratio of the determinants.

Using a conservative estimate of 0.016 for the standard deviation of sample proportions, we find that most estimators are significantly larger than the nominal size of 0.05 . Most strikingly, $\mathrm{L}_{0}$ mostly reject true null hypothesis implying that we need to a small sample adjustment for $\mathrm{L}_{0}$. Sargan (1980)'s Chi-squared test $\left(\mathrm{S}_{0}\right)$ is biased and overreject in most of the cases in finite sample except for the case when simultaneity parameters are $\beta=0.8, \delta=0.4$, and $\mathrm{T}=80$. F-form of Sargan's statistic $\left(\mathrm{F}_{0}\right)$ is less biased than $\mathrm{S}_{0}$ but properties of $\mathrm{F}_{0}$ resemble those of $\mathrm{S}_{0}$ as sample size increases. Hansen's GMM statistic $\left(\mathrm{G}_{0}\right)$ is also biased but nominal size of $\mathrm{G}_{0}$ approaches to the value of 0.05 while $\mathrm{S}_{0}$ departs significantly from $0.05 . \quad \mathrm{G}_{0}$ has the least bias for the nominal size.

\section{Asymptotic Power Of Four Statistics}

Figure 1 shows that, for most of the cases, estimated nominal power decreases as the econometric sample size increases and it increases with the increase of w. Asymptotic power is best approximated for $\mathrm{G}_{0}$ and then $\mathrm{F}_{0}$ while $\mathrm{S}_{0}$ is poorly approximated. 
Table 2: Response Surface Regressions

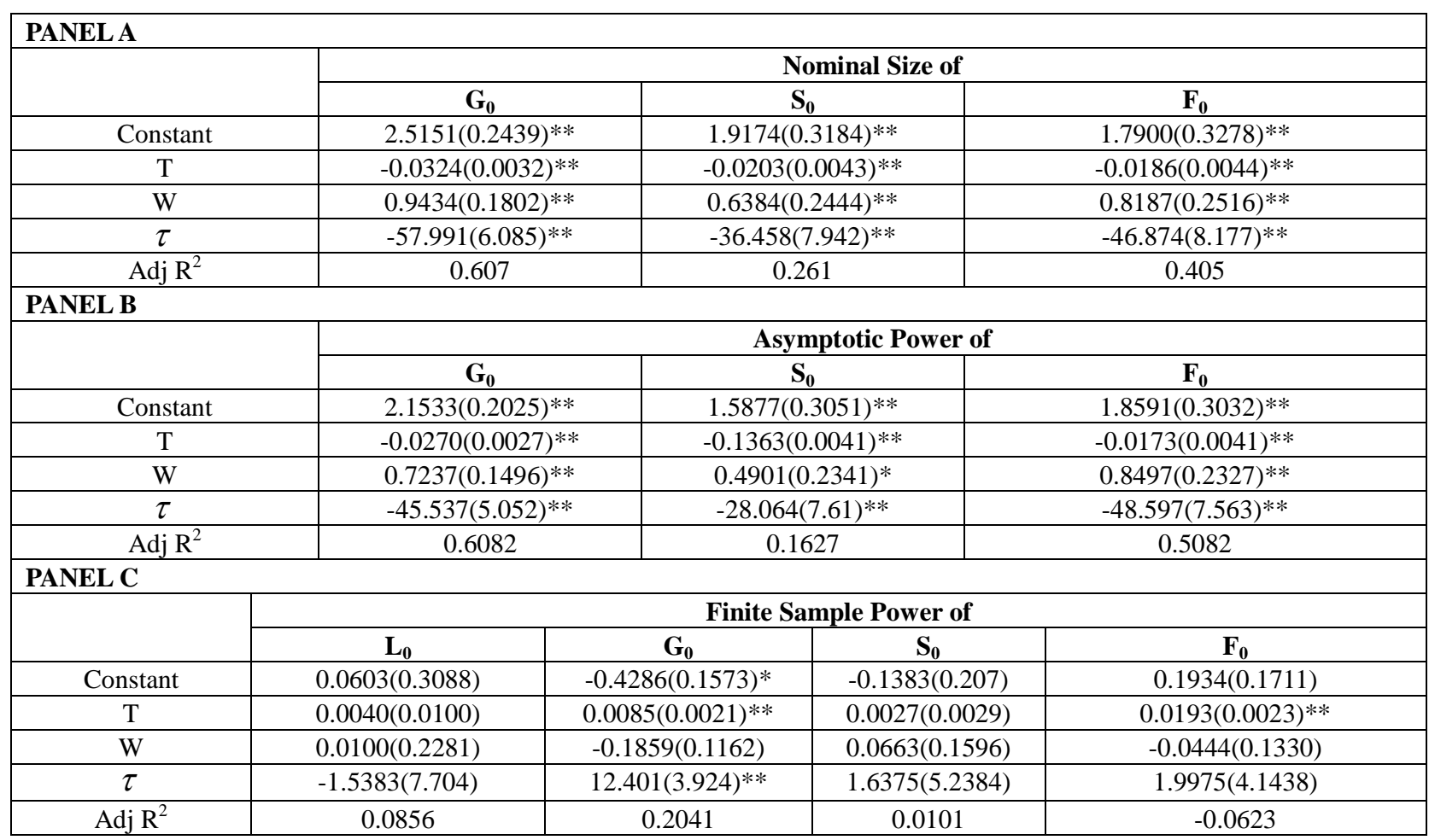

Note: Double asterisks denote significance at $1 \%$ critical level and single asterisk for $5 \%$.

$\mathrm{L}_{0}$ has rejection probability of one in most of the cases and $\mathrm{S}_{0}$ appears to be more powerful than $\mathrm{F}_{0}$ and $\mathrm{G}_{0}$ unless simultaneity parameter is large. Another interesting feature is that $\mathrm{S}_{0}$ outperform $\mathrm{F}_{0}$ even in small samples but $\mathrm{G}_{0}$ has the smallest rejection probability.

\section{Finite Sample Power Of Four Statistics}

In order to compare tests in terms of their power of a given size, the critical value for each test is set with reference to the empirical distribution of the statistic corresponding to the empirical size of 0.05 . In each replication, the false null is rejected if the test statistic exceeded the empirical critical value. Most of the estimates for the response surface regressions reported in Table 2 are insignificant and they poorly approximate the finite sample power of these statistics. However, for $\mathrm{G}_{0}$, the estimated coefficient of $\mathrm{T}$ is significant and finite sample power of $\mathrm{G}_{0}$ increases with the increase of $\mathrm{T}$. For all statistics, empirical power increase with the increase of sample size and empirical power decrease with the increase of $\mathrm{w}$ for most of the cases.

Out of four statistics, $\mathrm{L}_{0}$ is the most powerful test statistic in most of the cases and its finite sample power increase with the increase of the econometric sample size. $\mathrm{G}_{0}$ comes next and then $\mathrm{F}_{0}$ and $\mathrm{S}_{0}$. However, performance of $\mathrm{F}_{0}$ and $\mathrm{S}_{0}$ are quite similar. Estimated finite sample power is much smaller than its asymptotic power substantially. Most significant departure between asymptotic power and finite sample power comes for $S_{0}$ while $G_{0}$ approximates its asymptotic power well. Finite sample power of $\mathrm{L}_{0}$ is larger than other statistics and this increases with the increase of econometric sample size. 
Figure 1: Finite Sample Properties Of $L_{0}, G_{0}, S_{0}$, and $F_{0}$
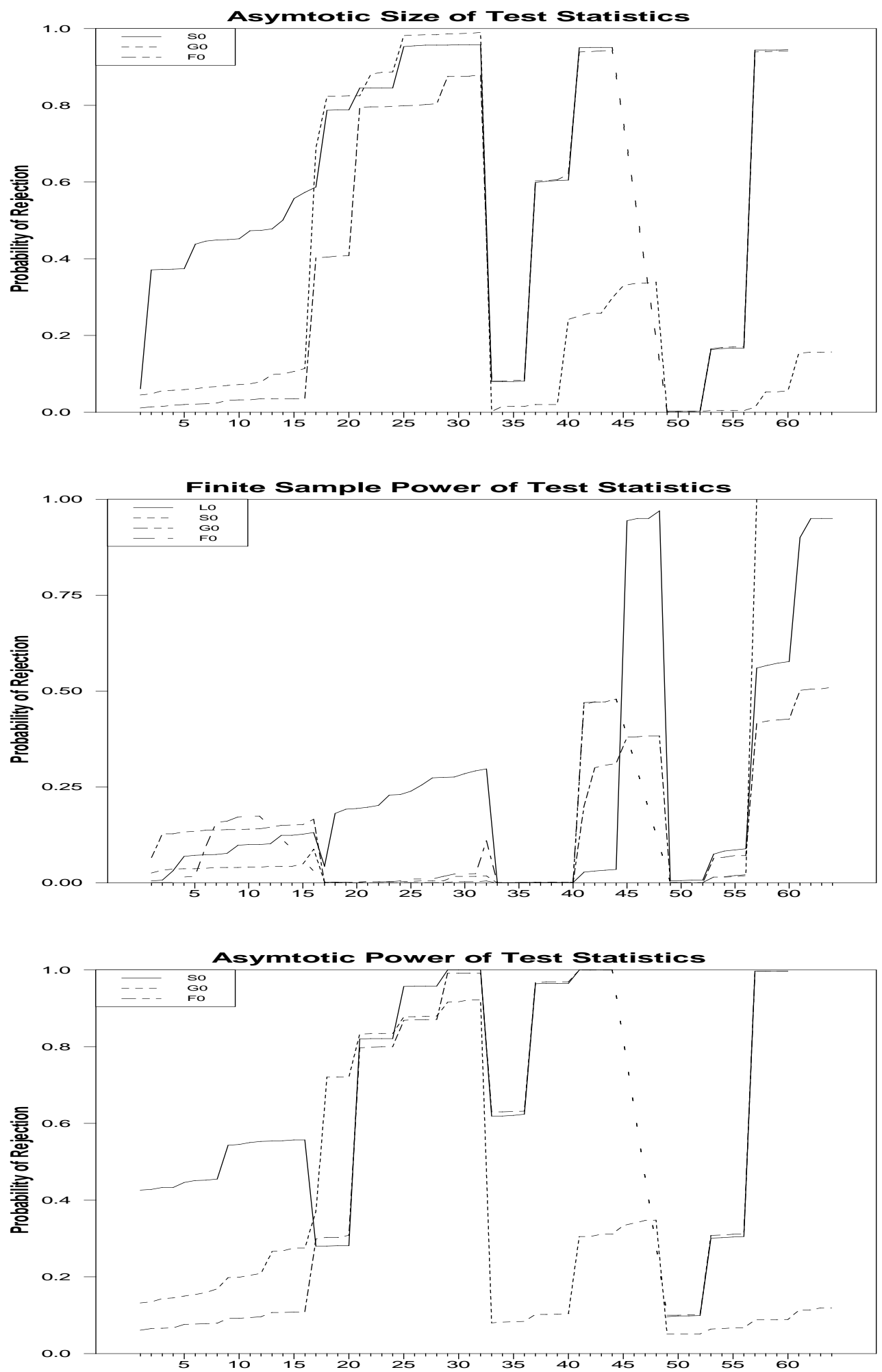


\section{CONCLUSIONS}

We conducted a Monte Carlo experiment to investigate the small sample properties of the four statistics which test the over-identifying restrictions in the simultaneous equations model. The likelihood-ratio test tends to reject the null hypothesis even when the errors in the model are consistent with the statistic's embodied hypothesis in the two equations model in finite sample. Small sample adjustment of Godfrey and Pesaran (1983) can be a solution for this bias. The problem seems less severe with test statistics of $\mathrm{S}_{0}, \mathrm{~F}_{0}$, and $\mathrm{G}_{0}$. The use of $\mathrm{G}_{0}$ helps a little but it is also biased in finite sample. F-form of Sargan's statistic $\left(\mathrm{F}_{0}\right)$ performs better than its Chi-squared form $\left(\mathrm{S}_{0}\right)$. Hansen's GMM statistic $\left(\mathrm{G}_{0}\right)$ has the smallest bias. Different methodology and software may allow more extensive design and more efficient simulation and control variate might help estimate $\pi_{T}$ more efficiently.

\section{REFERENCES}

1. Basmann, R. L., 1960, On Finite Sample Distributions of Generalized Classical linear identifiability Test Statistics, Journal of American Statistical Association, 55, 650-659.

2. Branson, W. H., Halttunen, H., and Masson, P., 1979, Exchange Rates in the Short-run: Some Further Results, European Economic Review, 12, 395-402.

3. Byron, R. P., 1974, Testing Structural Specifications Using the Unrestricted Reduced Form, Econometrica, $42,869-883$.

4. Ericsson, N. R., 1991, Monte Carlo Methodology and the Finite Sample Properties of the Instrumental variables Statistic for Testing Nested and Non-nested Hypothesis, Econometrica, 42, 869-883.

5. Godfrey, L. G. and Pesaran, M. H., 1983, Tests of Non-nested Regression Model: Small Sample Adjustment and Monte Carlo Evidence, Journal of Econometrics, 21, 133-154.

6. Harvey, A., 1990, The Econometric Analyses of Time Series, London:Phillip Allan.

7. Hansen, L. P., 1982, Large Sample Properties of Generalized Method of Moments Estimator, Econometrica, 50, 1029-1054.

8. Hendrey, D. F., 1984, Monte Carlo Experimentation in Econometrics, Chapter 16 in Handbook of Econometrics, volume 2, ed. By Grilliches and Intrilligatot, M. D., Amsterdam: North-Holland. 937-976.

9. Sargan, J. D., 1958, The Estimation of Economic Relationships Using Instrumental Variables, Econometrica, 26, 393-415.

10. Sargan, J. D., 1980, Some Approximations to the Distribution of Econometric Criteria Which are Asymptotically Distributed as Chi-squared, Econometrica, 48, 1107-1138. 